\title{
Frenectomía en el tratamiento de la anquiloglosia Reporte de un caso
}

Danuze Batista Lamas Gravina ${ }^{1}$ Caroline Fernandes da Costa $a^{2}$, Marilia Rodrigues Moreira ${ }^{3}$, Alexandre Franco Miranda $a^{4}$ Adriano Gonçalves de Castro ${ }^{5}$, Claúdia Maria de Souza Peruchi ${ }^{6}$

\section{Resumen}

Introducción: El frenillo lingual es una membrana mucosa adherida desde la base de la lengua hasta el reborde alveolar. Esta estructura en el recién nacido tiene una actuación importante en el acto de succión y amamantamiento. Un frenillo lingual corto influencia en la posición de los dientes en los arcos dentarios, en el amamantamiento y dificulta los movimientos de la lengua. Este aspecto patológico se llama anquiloglosia. Reporte de Caso: Este trabajo reporta el caso de una niña, 04 años de edad, que compareció con su padre a la Clínica de Odontopediatría de la Universidad Católica de Brasilia, con la queja de "lengua presa". Tras la anamnesis, se diagnosticó anquiloglosia. La niña presentaba el frenillo lingual adherido a la base de la lengua, presencia de diastema entre los incisivos centrales inferiores, lengua en formato de corazón cuando proyectada y dificultad para articulación de determinados fonemas. Luego de la firma del Formulario de Consentimiento informado por el responsable, se propuso como plan de tratamiento su remoción quirúrgica: Frenectomía. La cirugía fue realizada con éxito, con remoción de la membrana adherida, lo que le posibilitó a la niña una mejora inmediata en su habla. La niña fue encaminada para fonoaudiología para recuperar fonemas que no lograba pronunciar. Conclusión: La frenectomía le devolvió las funciones de la lengua al sistema estomatognático de la paciente, permitiendo la ejecución de sus funciones normales como alimentación, deglución y correcta articulación de palabras.

Palabras clave: Frenillo lingual, lengua presa, frenectomía, cirugía oral.

Relato de caso

\section{Frenulectomia no tratamento da Anquiloglossia - Relato de caso}

\section{Resumo}

Introdução: $\mathrm{O}$ freio lingual é uma membrana mucosa aderida desde a base da língua até o re- bordo alveolar. Esta estrutura no recém-nascido tem uma atuação importante no ato de sucção e amamentação. Um freio lingual curto influência na posição dos dentes nos arcos dentários, 
na amamentação e dificulta os movimentos da língua. Este aspecto patológico é chamado anquiloglossia. Relato de Caso: O presente trabalho relata o caso de uma criança, gênero feminino, 04 anos de idade, que compareceu com seu pai à Clínica de Odontopediatria da Universidade Católica de Brasília, com a queixa de "língua presa". Após a anamnese, diagnosticou-se anquiloglossia. A criança apresentava o freio lingual aderido à base da língua e presença de diastema entre os incisivos centrais inferiores. Metodologia: Após a assinatura do Termo de Consentimento livre e Esclarecido pelo responsável, foi proposto como plano de tratamento a remoção cirúrgica do mesmo - frenectomia. A cirurgia foi realizada com sucesso, com remoção da membrana aderida, o que possibilitou à criança uma melhora imediata na sua fala. A mesma foi encaminhada para fonoaudiologia para recuperar fonemas que antes não conseguia pronunciar. Conclusão: A frenectomia devolveu as funções da língua ao sistema estomatognático da paciente, permitindo a execução de suas funções normais como transporte de alimentos, deglutição e correta articulação de palavras.

Palavras chave: Odontopediatri, Freio Lingua, Tratamento.

\section{Review Article}

\section{Frenulectomia in the treatment of ankyloglossia- Case Report}

\section{Abstract}

Introduction: The lingual frenulum is a mucous membrane attached from the base of the tongue to the alveolar ridge. This structure in the newborn has an important role in sucking and breastfeeding. A short lingual frenulum influences the position of teeth in the dental arches, breastfeeding and hinders tongue movements. This situation is called ankyloglossia. Case Report: This study reports the case of a child, female, 04 years-old, who attended the Pediatric Dentistry Clinic of the Catholic University of Brasilia with her father, complaining of " tied-tongue". After the interview, was diagnosed with ankyloglossia. The child had the lingual frenulum adhered to the base of the tongue, making it difficult to pronounce certain phonemes. Surgical removal was proposed, and consent was given by parents for the frenectomy. The surgery was successful, allowing the child an immediate im- provement in speech. The same was referred for speech therapy to regain phonemes that were absent before the procedure. Conclusion: Frenectomy returned proper functions of the stomatognathic system of the patient, allowing the tongue to perform its duties as oral food transport, swallowing correct articulation of words.

Key Words: Lingual frenulum, frenectomy, oral surgery.

\section{Introducción}

Muchos aspectos de la boca de un bebé son únicos y peculiares a ese período de vida. En la infancia, la boca presenta desarrollo constante, estando en relación dinámica con otros sistemas y órganos, que también están en desarrollo. Presenta estructuras anatómicas únicas, transitorias y características de ese período de la vida. Así 
como los procesos fisiológicos son típicos, hay también algunas alteraciones de desarrollo y patologías que son propias de esa franja de edad. ${ }^{1}$

El impacto de las anomalías congénitas en la salud del individuo y en la familia es complejo, pues estas patologías son de naturaleza crónica y pueden interferir de forma negativa en todos sus aspectos. Además, los problemas médicos, psicológicos y económicos que la familia enfrenta son enormes, siendo imprescindible que los programas de salud incluyan estrategias de prevención dirigidas a los defectos congénitos. ${ }^{2}$

La anquiloglosia es considerada una anomalía congénita caracterizada por la inserción corta del frenillo lingual, identificada en diferentes franjas de edad, incluso en recién nacidos. ${ }^{3}$ Individuos con esta anomalía, dependiendo de la edad, pueden presentar dificultades en la fonación, masticación, deglución atípica, postura anormal de la lengua, amamantamiento, diastema entre los incisivos centrales inferiores, irritación del frenillo lingual o periodontopatias. ${ }^{4-9}$

Los audiólogos encuentran muchos pacientes con diferentes quejas que llevan a la hipótesis de que el frenillo de la lengua puede estar con alguna alteración siendo el causante de los problemas, o por lo menos, siendo su agravante. Los síntomas más comunes que pueden generar tales hipótesis son: imprecisión de la articulación del habla; cambio de fonema por otro o con distorsión; pequeña apertura de la boca durante el habla; imprecisión o ineficacia de los movimientos de la lengua en movimientos aislados; forma de corazón en el ápice de la lengua cuando es protraída, lengua con poca protrusión, o aún con protrusión conllevando su ápice hacia abajo; lengua con postura en el piso de la boca; dificultades de hacer movimientos con la pun- ta de la lengua; dificultad de succión durante el amamantamiento; masticación ineficiente y deglución con alteración por dificultad de acoplamiento de la lengua en el paladar duro, entre otras. ${ }^{9}$

En la literatura, hay diferentes tentativas de clasificaciones para describir las alteraciones del frenillo: lengua presa, anquiloglosia, frenillo hipertrófico, frenillo espeso, frenillo muscular, frenillo fibrótico, frenillo con inserción anteriorizada, frenillo corto y frenillo con inserción anteriorizada y corto. ${ }^{10} \mathrm{El}$ frenillo lingual es una membrana mucosa adherida desde la base de la lengua hasta el reborde alveolar. Esta estructura en el recién nacido tiene una actuación importante en el acto de succión y amamantamiento. Un frenillo lingual corto dificulta los movimientos de la lengua y puede presentar bastante adherido al piso de la cavidad bucal. Este aspecto patológico se llama anquiloglosia ${ }^{10}$. Este desvíación de normalidad presenta un carácter hereditario, dictado por una herencia autosómica dominante, y su frecuencia puede ser influenciada por aislamiento físico y cultural ${ }^{11}$

El frenillo lingual o frenillo de la lengua está conectado al piso de la boca que permite movimiento libre de la lengua. No se trata de tejido muscular, sino un pliegue mediano de túnica mucosa que pasa de la encía para el lado posteroinferior de la lengua y recubre el lado lingual de la cresta alveolar anterior. Está formado por tejido conjuntivo fibrodenso y, muchas veces, por fibras superiores del músculo geniogloso. Cuando ocurre el desarrollo y crecimiento óseo con prolongación lingual y erupción dentaria, el frenillo lingual migra hacia la posición central hasta ocupar su posición definitiva con el nacimiento de los dientes. ${ }^{10}$

Se clasifica el frenillo lingual en corto, cuando él se presenta en fijación anteriorizada, es decir, 
cuando la inserción en la faz sublingual ocurre en cualquier punto tras el medio de la faz sublingual hasta la punta de la lengua. ${ }^{12}$ Madeira $(1993)^{13}$ describe el frenillo como una parte de la mucosa oral que forma una dobla ondulada determinando una pliega franjada. Esa estructura recubre la vena profunda de la lengua y la glándula lingual anterior cerca del ápice. Histológicamente, el frenillo lingual está compuesto por un tejido conjuntivo rico en fibras colágenas y elásticas, con algunas fibras musculares, vasos sanguíneos y células engrasadas, y recubierto por un epitelio pavimentoso estratificado. ${ }^{14}$

La anquiloglosia, conocida como lengua presa en su forma popular, constituye una anomalía del desarrollo caracterizada por alteración en el frenillo de la lengua que resulta en limitaciones de los movimientos de esa estructura, que puede generar cambios en el habla y deglución. El cambio de la inserción sucede de la punta de la lengua hasta el reborde alveolar lingual y es visible ya en el nacimiento. ${ }^{7}$ Su definición va desde una imprecisa descripción de lengua que funciona con la extensión de la actividad menor que la normal hasta la descripción de frenillo corto, espeso, muscular o fibroso. ${ }^{15}$

La fusión de la lengua con el piso de la boca se muestra una condición rara; la anquiloglosia parcial es más común. Esa anormalidad dificulta los movimientos de la lengua, principalmente en la pronuncia de ciertas consonantes y diptongos labiodentales. A pesar de ser una entidad clínica bastante reconocida, la anquiloglosia en niños menores de 1 año representa un desafío cuanto a su diagnóstico para los cirujanos dentistas. ${ }^{15}$ Además, ella interfiere también en el proceso de cepillado $\mathrm{y}$, por consiguiente, favorece riesgo de acúmulo de placa, instalación de inflamación tisular y retracción de encías. ${ }^{16}$
En estudio realizado en población indígena brasileña, la anquiloglosia fue observada en 108 $(37,11 \%)$ de los indígenas examinados. La mayor prevalencia fue encontrada en el sexo masculino con 57 casos $(43,8 \%)$, mientras el sexo femenino presentó $51(31,7 \%)$ de los casos. La distribución de la franja de edad fue hecha tras análisis de clasificación jerárquica, distribuidas en tres grupos: 1-20 años, 21-45 años y 46-99 años y la anquiloglosia presentó mayor prevalencia en la franja de edad de 1- 20 años, con 65 casos (40,6\%). ${ }^{17}$

Según el criterio de diagnóstico utilizado y de la población estudiada, la prevalencia puede variar de 1 a 10,7\%. No hay uniformidad de definición diagnóstica y la mayoría de los datos en la literatura son generalmente, provenientes de serie de casos o relatos de casos. Parece que hay predilección por el sexo masculino. ${ }^{18}$

En un estudio conducido en la Universidad Federal de Santa Maria, Brasil, fueron triados ${ }^{1}$. 516 pacientes, con edades entre 5 y 16 años, de ambos los sexos. El diagnóstico de anquiloglosia fue realizado por un odontopediatra, a través de examen clínico. Tras el diagnóstico, los pacientes eran encaminados para las evaluaciones fonoaudiológica y ortodóntica. De los pacientes evaluados, 21 presentaron diagnóstico de anquiloglosia, siendo que hubo prevalencia en el sexo masculino y en las franjas de edad de los 5 a los 6 años y de los 11 a los 12 años. La mayoría presentó alteraciones en la postura y movilidad lingual, así como en las funciones de deglución y fonoarticulación. ${ }^{18}$

En el estudio de Chaves ${ }^{19}$, en que se verificó la relación entre lactancia materna y la presencia de frenillo lingual alterado, se encontró una prevalencia de $4,1 \%$, variando significativamente con estudios anteriores. 
Hay muchas maneras de tratamiento, incluyendo técnicas quirúrgicas convencionales y el tratamiento con láser. Frenotomia es la sencilla escisión del frenillo; frenectomia es completa escisión, donde se remueve toda la membrana y la frenuloplastia envuelve varios métodos para reposicionar la lengua presa y corregir la situación anatómica. ${ }^{20,21}$

El tratamiento quirúrgico puede ser una opción y debe ser realizado tras cuidadosa evaluación clínica y fonoaudiológica e instituido cuando trastornos fisiológicos, patológicos e/o fonoaudiológicos relacionados a la anquiloglosia estén presentes y justifiquen tal procedimiento. ${ }^{7}$ En este caso, la remoción de un segmento de la cinta fibrosa del frenillo (frenectomía) o sólo la sección de este (frenotomía) debe ser realizado. $^{5}$

\section{Reporte de caso}

Una niña, 4 años de edad, compareció con su padre a la Clínica de Odontopediatría de la Universidad Católica de Brasília, con la queja de "lengua presa". Tras la anamnesis, se diagnosticó anquiloglosia. La niña presentaba el frenillo lingual adherido a la base de la lengua,

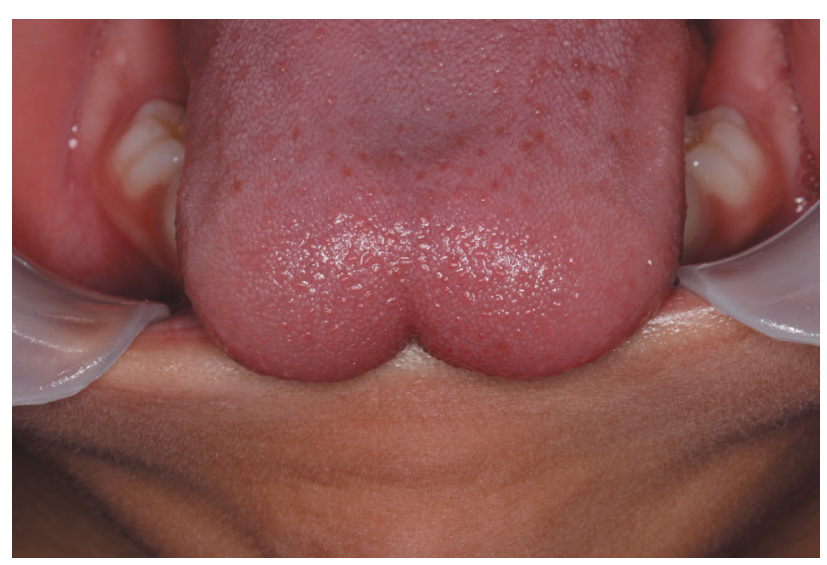

Figura 1. Lengua en forma de corazón cuando es proyectada.

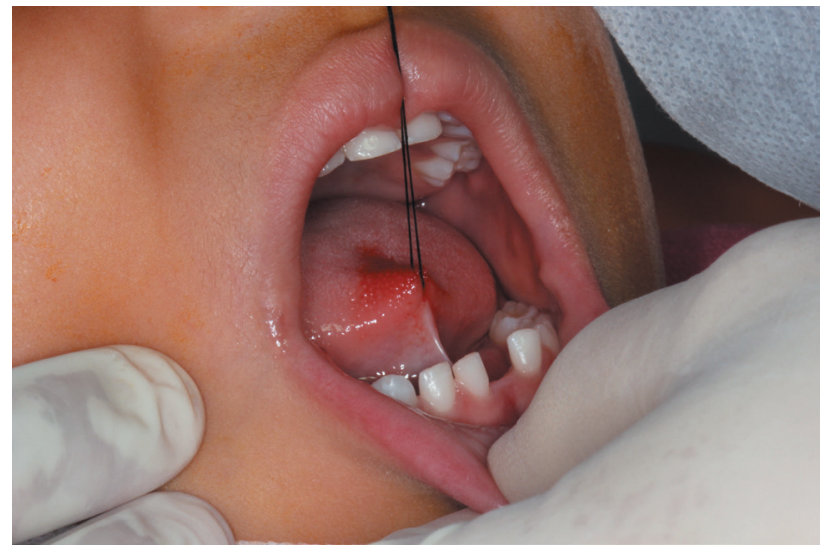

Figura 2. Transfijación de hilo de sutura de seda en la extremidad de la lengua y diastema presente ente incisivos inferiores.

lo que dificultaba el habla y los movimientos de la lengua (Fig. 1) y presencia de diastema entre los incisivos inferiores (Fig 2). Fue propuesto como plan de tratamiento su remoción quirúrgica por medio de frenectomía.

Inicialmente, fue hecha la técnica de manejo, seguida de antisepsia extra e intrabucal, con clorexidina $0,12 \%$ y para extra bucal Iodopovidona (PVPI). Tras el secado de la mucosa, fue hecha la aplicación del anestésico tópico, seguida de bloqueo del nervio lingual y alveolar inferior y en la región del frenillo lingual con lidocaína a $2 \%$ y epinefrina 1 : 100.000. Tras la confirmación de la anestesia, a través de las señales y testes, fue realizada la transfijación de la lengua con hilo de sutura seguido de incisión en forma de rombo (Fig 3). Después, fue removido el tejido que prendía la lengua al piso bucal. Por fin, se realizó la sutura en 5 puntos aislados (Fig 4). Recomendaciones postoperatorias y prescripción de analgésico fueron repasadas al responsable por la paciente. Una semana después, fue hecha la remoción de la sutura, prescripta medicación Omcilon-A orabase ${ }^{\circledR}$ y aplicación de clorhexidina $0,12 \%$ debido a la ulceración local presente. Controles periódicos 


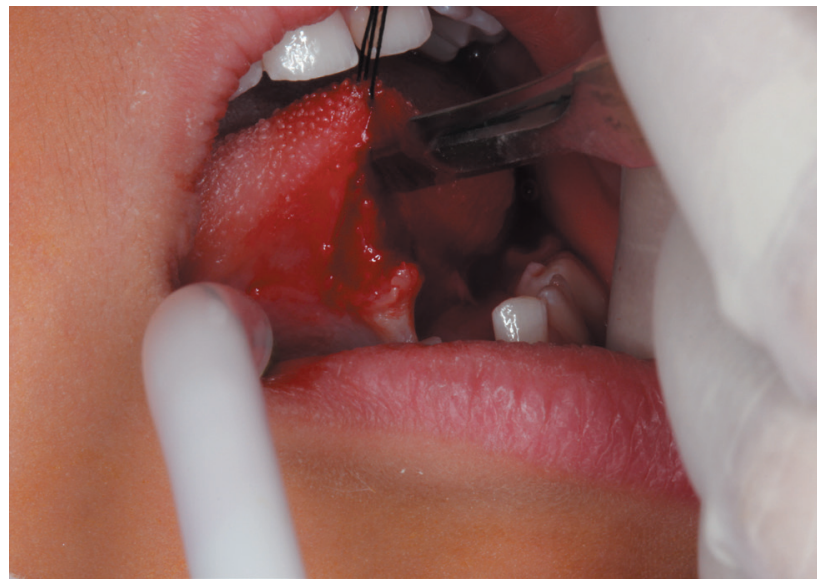

Figura 3. Seccionamiento del frenillo lingual en su porción mediana.

fueron realizados con la finalidad de identificar si el problema fue solucionado.

\section{Discusión}

La corrección de la anquiloglosia en una edad precoz reduce el riesgo de complicaciones a los lactantes, y la frenectomía debe ser ejecutada cuando hay interferencia en la deglución y en el habla ${ }^{17}$. Bebés con frenillo de la lengua alterado pueden tener problemas en el afianzamiento del seno, dificultando la succión de leche e interfiriendo en la ganancia de peso. La liberación quirúrgica del frenillo, cuando cri-

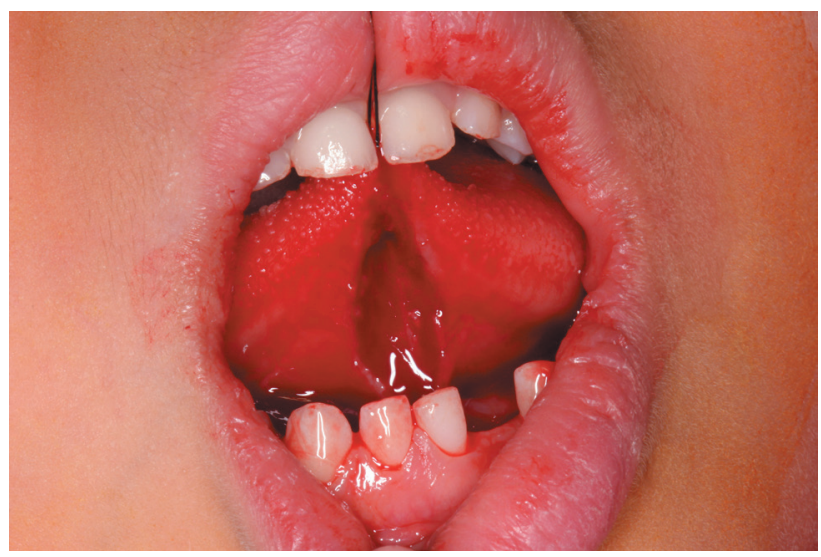

Figura 4. Remoción del frenillo lingual con bisturí, incisión en forma de rombo, divulsión de la lengua. teriosamente indicada, promueve la mejora de esa función.

La paciente relatada tuvo el diagnóstico de forma precoz, pero no logró el tratamiento debido a la gran lista de espera en los hospitales de la red pública. Hoy, Brasil ofrece el test de la lengua en todas las maternidades, abriendo más un campo de actuación para los profesionales de la salud y beneficiando la población desde que ese examen pasó a ser obligatorio a través de la ley en el 13.002, de 20 junio de 2014. La presente paciente no se benefició de esa ley y tuvo su habla perjudicada y problemas sociales durante los 4 primeros años de vida. Por pertenecer a una clase social y económicamente desfavorecida, no logró realizar el procedimiento quirúrgico en la red privada, aguardando una plaza en el servicio público, lo que fue imposibilitado por cuestiones diversas.

El pediatra, el odontopediatra y el clínico general son los profesionales capacitados para detectar anormalidades en la boca de recién nacidos, lactantes y niños. Cabe al pediatra el diagnóstico de las manifestaciones presentes en la boca de los bebés en el inicio de la vida. El odontopediatra, generalmente examina los niños a partir del período de la erupción de los dientes primarios (sobre los 6 meses de edad). Hoy, con el advenimiento de la odontología para bebés, la atención precoz antes del nacimiento de los dientes volvió posible el diagnóstico de alteraciones bucales, como la anquiloglosia. La evaluación de problemas en el frenillo lingual permite la identificación de anomalías de su inserción y prevención de alteraciones de las funciones de deglución y habla por la corrección quirúrgica de la anquiloglosia.17 Esta paciente, con 04 años de edad, a pesar de estar con la indicación quirúrgica hace algún tiempo, aún aguardaba en la 
lista de espera de red pública de salud. A través de sondeos hechos por los estudiantes del curso de odontología de la Universidad, la niña fue identificada con el problema y encaminada para el tratamiento en la facultad de Odontología.

No hay dudas de que cualquier problema que comprometa la salud de la lengua puede reflejar seriamente en las funciones bucales. La lengua posee función importante en el transporte de alimentos y en la deglución, así como papel esencial en la articulación de las palabras. Ella tiene influencia en la posición de los dientes en los arcos dentarios y en el amamantamiento.17

En el caso relatado, la anquiloglosia presentaba relevancia clínica y social, ya que estaba provocando alteraciones morfofuncionales. La niña exhibía dificultad en el habla, limitación de los movimientos de la lengua, deglución anormal a causa del frenillo lingual corto. Ella sentía también dificultad en la articulación de palabras con fonemas labiodentales, así como la presencia de diastema entre os incisivos centrales inferiores.

El diagnóstico clínico inicial de la anquiloglosia puede ser hecho basado en la dificultad que el paciente presenta en tocar con la lengua en el paladar duro y proyectarla más allá de los incisivos inferiores. En el caso presentado, la lengua, cuando proyectada, presentaba la forma de corazón, característica de la anquiloglosia. Otra manera de diagnóstico puede ser la mediciónn del frenillo lingual, utilizando calibrador. Pocos estudios procuran evaluar el frenillo a través de medidas cuantitativas. Probablemente esto ocurre por la dificultad en medir tejidos blandos, ya que imprecisiones podrán ser frecuentes.9 En el estudio de Lee22, los frenillos linguales fueron con una regla creada para esta finalidad y clasificados de la siguiente manera: longitud medio del frenillo con menos de que $10 \mathrm{~mm}$ sería una anquiloglosia suave; entre 10 y $15 \mathrm{~mm}$ moderada; más que $15 \mathrm{~mm}$ anquiloglosia severa tipo 1 y por último fue clasificado como anquiloglosia severa tipo 2 aquellos frenillos que clínicamente eran clasificados como severa anquiloglosia aunque tuviesen menos de $15 \mathrm{~mm}$ de longitud. La paciente se inscribía en la clasificación moderada, con un frenillo que mide sobre $12 \mathrm{~mm}$.

Se sabe que el diagnóstico de la presencia o no de anquiloglosia puede estar asociado a la manera como el examinador define esta anomalía de desarrollo, pues, dependiendo de los criterios adoptados, o el frenillo de un individuo puede ser caracterizado como normal o como alterado. ${ }^{18}$ El diagnóstico de la paciente fue de frenillo alterado, por esto se optó por la realización de la cirugía. En función de la clasificación moderada, se optó por la cirugía convencional, técnica utilizada en la facultad de odontología de la Universidad Católica de Brasilia.

En relación con los niños portadores de esta anomalía, el seguimiento clínico es fundamental, pues permite observar la regresión o permanencia de la anquiloglosia a lo largo de la edad, así como evaluar mejor la necesidad de intervención quirúrgica. ${ }^{19}$ En este caso, no ocurrió la regresión del frenillo, siendo, de esta manera, indicado el tratamiento quirúrgico.

El aumento en la incidencia de caries también puede ser considerado como una complicación en relación a la existencia de la anquiloglosia, en función del aumento en la dificultad con autolimpieza en dientes posteriores. Sin embargo, esta consideración debe ser hecha, sólo, cuando factores dietéticos e higiene bucal deficiente no son identificados como factores asociados a la enfermedad caries. La presente paciente, 
presentaba todos los dientes sanos y según su padre, ella presentaba buenos hábitos de higiene bucal y dieta. Otra alteración frecuente es la presencia de bolsa periodontal en que la inserción alta del frenillo promueve la retracción de las encías, lo que facilitaría la acumulación de placa y consecuentemente la formación de bolsa periodontal. ${ }^{19}$ Esta relación tampoco no fue encontrada en el caso relatado.

El tratamiento quirúrgico de la anquiloglosia permite la corrección inmediata de algunas de las alteraciones presentes. En este caso, algunas funciones como liberaciones de los movimientos de la lengua fueron percibidas inmediatamente tras la cirugía. Algunos problemas, antes exis- tentes, como dificultad de pronuncia de determinados fonemas sólo presentarán mejora tras un seguimiento fonoaudiológico.

\section{Conclusión}

En el caso presentado, la inserción anormal de la lengua alteraba de modo significativo las funciones de deglución, los movimientos de la lengua, el habla y la articulación de las palabras de la paciente. Con la frenectomía lingual, parte de las funciones de la lengua fueron restablecidas, una vez que la paciente aún se encuentra en tratamiento fonoaudiológico para recuperar y aprender fonemas que antes no lograba pronunciar.

\section{Referencias bibliográficas}

1. Baldani MH, Lopes CMDL, Scheidt WA. Prevalência de alterações orais em crianças atendidas na clinica odontológica da rede publica de Ponta Grossa -PR, Brasil. Pesqui Odontol Bras. 2001; 15( 4):302-07.

2. Emond A, Ingram J, Johnson D, Blair P, Whitelaw A, Copeland M, and Sutcliffe A. Randomised controlled trial of early frenotomy in breastfed infants with mild-moderate tongue-tie. Arch Dis Child Fetal Neonatal Ed. 2014 May; 99(3): F189-F195. Published online 2013 Nov 18.doi:10.1136/archdischild-2013-305031

3. Messner AH, Lalakea L. The effect of ankyloglossia on speech in children. Otolaryngol Head Neck Surg. Dec 2002; 127(6): $539-45$.

4. Pozza DH, Deyl JT, Cardoso ES, Cançado RP, Oliveira MG. Frenulectomia lingual: revisão da literatura e relato de caso clínico. Rev Odontol. UFES mai-aug 2003; 5 (2): 19-25

5. Segal LM, Stephenson R, Dawes M, Feldman P. Prevalence, diagnosis, and treatment of ankyloglossia: Methodologic review. Can Fam Physician. 2007; 53 (6): 1027-33.

6. Geddes DT, Kent JC, Mc Clellan HL, Garbin CP, Chadwick LM, Hartmann PE. Sucking characteristics of successfully breastfeeding infants with ankyloglossia: a case series . Acta Pædiatrica 2010; 99 ,301-3.

7. Brito SF, Marchesan IQ, Bosco CM, Carrilho ACA, Rehder MI. Lingual frenulum: classification and conduct according to speech language pathologist, odontologist and otorhinolaryngologist perspective. Rev CEFAC. São Paulo, 2008 ; 10(3):343-51. 8. Power RF, Murphy JF. Tongue-tie and frenotomy in infants with breastfeeding difficulties: achieving a balance. Arch Dis Child. 2014;99:Suppl 2 A502.

9. Marquesan IQ. Lingual frenulum: quantitative evaluation proposal. The Internatational Journal of Orofacial Myology. São Paulo, 2005; 31: 39-48.

10. Melo G, Isa FI, Melo NSFO. Anquiloglossia: prevalência entre crianças de 0 a 18 meses atendidas em uma unidade de saúde do município de Colombo. Revista Dens. 2007;15(2). 
11. Jamieson LM, Bailie RS, Beneforti M, Koster CR, Spencer AJ. Dental self-care and dietary characteristics of remote-living Indigenous children. Rural and Remote Health. 6: 503 (online), 2006. http://www.rrh.org.au/publishedarticles/article_ print_503.pdf

12. Braga LAS, Pantuzzo CA, Motta AR. Prevalence of change in frenulun lingual and its implications in speech of school children. Rev CEFAC. 2009;11(Supl3):378-90.

13. Madeira MC. Anatomia da Face. Bases anátomo-funcionais para prática odontológica. São Paulo : 8 ed ; Ed Sarvier ; 2013 cap 1.

14. Guedes-Pinto AC. Cirurgia em odontopediatria. In: Odontopediatria. 9. ed. São Paulo: Livraria Santos; 2016. p. 441

15. Kotlow L. Ankyloglossia (tongue-tie): a diagnostic and treatment quandary. Quintessence Int.1999; 30(4):259-62.

16. Yared KFG, Zenobio EG, Pacheco W. A etiologia multifatorial da recessão periodontal. R Dental Press Ortodon Ortop Facial. 2006;11 (6):45-51.

17. Vieira EMM, Salineiro FS, Hespanhol D, Musis CR, Jardim Junior EG, Frequency of ankyloglossia in a community of native Brazilians. RGO - Rev Gaúcha Odontol. 2010; 58(2): 215-18.

18. Morisso MF, Berwig LC, Silva AMT. Ankyloglossia- Related Changes In The Stomatognathic System. RGO, Rev Gaúch. Odontol. 2012; 60 (2) 203-8.

19. Chaves JC, Silva FR, Breda A, Rodrigues AH, Fujinaga. Aleitamento materno e frênulo lingual: estudo de prevalência. Disponible en http://inesco.org.br/conferencias/index.php/2congresso/2cpsp/paper/view/345

20. Melo NSFO, Lima AAS, Fernandes A, Silva RPGVC. Anquiloglossia: relato de caso. RSBO. 2011; 8 (1):102-7.

21. Barot VJ, Vishnoi SL, Chandran S, Bakutra GV. Laser: The torch of freedom for ankyloglossia. Indian J Plast Surg. $2014 ; 47$ (3): 418-22.

22. Lee SK, Kim YS, Lim CY. A pathological consideration of ankyloglossia and lingual myoplasty. Taehan Chikkwa Uisa hyophoe Chi. 1989; 27(3):287-308.

Recibido: 06-06-2015

Aceptado: 04-01-2016

Correspondencia: Danuze Batista Lamas Gravina;

SQSW 305 Bloque H piso 605 - Sudoeste -

Brasília - DF, Código postal- 70673428 - Brazil

Correo electrónico-danuzeblamas@globo.com 